\title{
Evaluation of the first experience with COVID-19: An observational study on the screening policy among employees and patients of a Dutch teaching hospital
}

\author{
Veronica Weterings ( $\square$ Vweterings@amphia.nl ) \\ Amphia Ziekenhuis https://orcid.org/0000-0003-1101-4494 \\ Miranda van Rijen \\ Amphia Ziekenhuis \\ Mark Tinga \\ Amphia Ziekenhuis \\ Job Meijs \\ Amphia Ziekenhuis \\ Wouter van den Bijllaardt \\ Amphia Ziekenhuis \\ Jan Kluytmans \\ Amphia Ziekenhuis
}

\section{Research}

Keywords: COVID-19, SARS-CoV-2, corona virus, healthcare workers, hospital-onset, nosocomial transmission

Posted Date: June 9th, 2020

DOI: https://doi.org/10.21203/rs.3.rs-33375/v1

License: (c) (1) This work is licensed under a Creative Commons Attribution 4.0 International License. Read Full License 


\section{Abstract}

\section{Background}

In the beginning of March 2020, the Amphia hospital in Breda was one of the first hospitals in the Netherlands that received patients with COVID-19. Within ten days after the first COVID-19 positive patient, the number of (suspected) COVID-19 cases among patients and employees increased rapidly and thus the number of requests for SARS-CoV-2 PCR tests. From mid-March, we changed to a non-test-based strategy for employees whereby a work ban was implemented, based on the presence or absence of respiratory symptoms without the use of a diagnostic test. The objective of our study was to evaluate the safety of the non-test-based strategy.

Methods

The study period was March 1 to May 22, 2020. For all patients in whom the SARS-CoV-2 PCR test was positive $>2$ days after admission, the medical record was reviewed by infection control staff for (respiratory) symptoms, laboratory results and lung CT/X-ray images to assess possible hospital-onset COVID-19. For employees, the number of sick reports per week were extracted from the department of Human Resources. Reason for reporting sick was not registered.

Results

Ten of the 374 patients (2.7\%) developed symptoms suspected for COVID-19 $\geq 3$ days after admission and were tested positive for SARS-CoV-2, of which nosocomial transmission is the most likely cause in three patients.

In the period in which the employees were still actively tested for SARS-CoV-2, there was a temporary increase in the number of sick reports (week 10-11). After this two-week period, the number of sick reports decreased rapidly. The total number of sick reports in the first 20 weeks of 2020 is comparable or lower than the total number of sick reports in the same period in previous years.

Conclusion

With this non-test-based strategy for employees combined with a restriction of visitors to the patients and strict isolation of COVID-19 (suspected) patients, there was no excessive absenteeism of work due to illness and there were only few patients with indications of COVID-19 acquisition in the hospital.

\section{Background}

As the COVID-19 pandemic spreads in the population and healthcare facilities across the globe, prevention of nosocomial COVID-19 infection among hospital employees and patients is challenging. 
The Amphia hospital in Breda was one of the first hospitals in the Netherlands that was confronted with COVID-19. On March 2 2020, the first admitted patient with COVID-19 was diagnosed. Within ten days after the first COVID-19 positive patient, the number of (suspected) COVID-19 cases among patients and employees increased rapidly and thus the number of requests for SARS-CoV-2 PCR tests. Initially both patients and employees with symptoms of COVID-19 were tested. Due to shortage of testing capacity and increasing turnaround time, it was decided to use COVID-19 diagnostics primarily in patient care and stop the testing of employees (Fig. 1).

From that moment on the presence of symptoms was used to decide if employees were allowed to work. The objective of our study was to evaluate the safety of the non-test-based strategy.

\section{Method}

An observational study was performed in the Amphia hospital Breda, a large teaching hospital with approximately 4,500 employees in the south of the Netherlands. The study population consisted of hospitalised patients who were tested positive for COVID-19 and all employees. The study period was March 1 to May 22, 2020.

For all patients in whom the SARS-CoV-2 PCR test was positive $>2$ days after admission, the medical record was reviewed by infection control staff for (respiratory) symptoms, laboratory results and lung CT/X-ray images to assess possible hospital-onset COVID-19. We defined patients with hospital-onset COVID-19 as those admitted to our hospital for a reason other than acute respiratory infection in whom respiratory symptoms or fever developed $>2$ days after admission and COVID-19 infection was confirmed using SARS-CoV-2 PCR test. For employees, we extracted the number of sick reports per week from the department of Human Resources. Reason for reporting sick was not registered.

\section{Testing for COVID-19}

A semi-quantitative real-time reverse-transcriptase PCR (RT-PCR, 45 cycles) targeting the E-gene was performed as described previously ${ }^{1}$ on oropharyngeal samples or other respiratory samples for patients and on self-collected oropharyngeal samples for employees.

\section{Policy for testing of patients}

All patients with fever and/or symptoms of respiratory infection were identified on admission and tested for COVID-19. Based on the perceived risk of a COVID-19 infection by the attending clinician patients were pre-emptively placed in either contact-droplet isolation precautions (low risk) or strict isolation precautions (high risk or proven COVID-19). In case of contact-droplet precautions, patients were placed in a single room and HCWs donned gloves, eye protection, apron and a type IIR surgical facemask upon entry. When strict isolation was performed, patients were placed in a room with an anteroom and negative air pressure. All healthcare-workers donned gloves, eye protection, gown with long sleeves, cap and a mask before entering the patient room. The type of mask for strict precautions depended on the 
procedure: when performing aerosol-forming procedures a FFP2 respiratory mask was worn, otherwise a type IIR surgical facemask was worn. From mid-March, the influx of COVID-19 (suspected) patient increased rapidly and cohorted wards for highly suspected or proven COVID-19 patients were established maintaining strict isolation measurements except for the room with anteroom and negative air pressure.

\section{Policy for employees}

From March 3, all employees were asked to measure their temperature twice a day and monitor onset of respiratory symptoms. An employee with fever $\left(>38^{\circ} \mathrm{C}\right)$ and respiratory symptoms and/or systemic symptoms (muscle pain, malaise) was banned from work after taking an oropharyngeal sample for PCR testing. Employees with only coughing symptoms continued work pending the PCR test result. When tested positive for SARS-CoV-2, the employee stayed at home until symptom-free for 24 hours. After March 13, we changed to a non-test-based strategy for employees whereby a work ban was implemented, based on the presence or absence of respiratory symptoms without the use of a diagnostic test, as shown in a flow chart (Fig. 1). Employees were allowed to resume work when symptoms had resolved for 24 hours.

\section{Results}

The prevalence of COVID-19 in employees has already been published ${ }^{2}$. About $5 \%$ of the employees with respiratory symptoms were tested SARS-CoV-2 positive, often with only mild symptoms, and a number of employees had worked with these symptoms. Figure $2 a$ shows the number of sick reports per week. In the period in which the employees were still actively tested for COVID-19, there was a temporary increase in the number of sick reports (week 10-11). After this two-week period, the number of sick reports decreased rapidly. Furthermore, the total number of sick reports in the first 20 weeks of 2020 is comparable or lower than the total number of sick reports in the same period in previous years (Fig. 2b)

Besides the higher absenteeism from work, another potential adverse effect of not testing employees can be the risk of COVID-19 positive employees who infect patients in the hospital. As shown in Fig. 3, hardly any patients developed a hospital-onset COVID-19 infection. Until May 22, 2020, 374 COVID patients who tested positive for SARS-CoV-2 had been hospitalised. In total, 364 patients (97.3\%) had symptoms suspected for COVID-19 infection upon admission. Ten of the 374 patients (2.7\%) developed symptoms suspected for COVID-19 $\geq 3$ days after admission and were tested positive for SARS-CoV-2: four patients developed symptoms on days three after admission, which is within the median incubation period of COVID-19 of five days ${ }^{3}$. These infections are considered most likely to be acquired in the community. Two patients developed symptoms on respectively day six and seven after admission, allowing both COVID-19 acquisition within and outside the hospital as a possibility. Four patients developed symptoms more than seven days after admission on respectively day $8,13,10$ and 44 . One of these patients had been visited by a family member with respiratory symptoms. We were not able to find a reasonable explanation for the acquisition for the other three patients and were considered to be to be acquired in the hospital. All patients were discharged in relatively good health. 


\section{Discussion}

Our observational study indicates that the policy of not routinely testing employees is safe for both patients and employees. In addition, it was not associated with an excessive absenteeism from work. Therefore, we decided to continue this policy now the testing capacity is sufficient to test both patients and employees. In special cases where the deployment of the employee has direct consequences for the continuity of a critical process, a test can be considered. However, this is only sporadically indicated. During this study, there were other control measures in place that may have contributed to the findings. First, the amount of visitors to patients admitted to the hospital was strongly reduced. Also the outpatient clinics were only open to those requiring urgent medical care. Last, it should be noted that the building were the Amphia hospital is located is newly constructed and was opened in 2019 and only has single rooms with private sanitary facilities.

In addition, our study has some limitations. First, there was no follow-up period after discharge, which may lead to missed cases of nosocomial transmission in patients - in case symptoms of COVID-19 arose after discharge. Secondly, we do not have any information on the reason for calling in sick for employees and therefore it is not clear how many sick reports were due to COVID-like symptoms.

\section{Conclusion}

This study supports the safety of hospital care during the COVID-19 pandemic when an active screening policy for patients and healthcare workers is instituted. The symptom-based strategy for health care workers did not result in a high absenteeism from work and hospital-onset COVID-19 infections were observed rarely. We recommend other healthcare institutions that want to implement this policy to monitor the outcome in a comparable way to ensure the safety of the patients and health care workers.

\section{Abbreviations}

COVID-19

Coronavirus disease 2019

SARS-CoV-2

Severe acute respiratory syndrome coronavirus 2

FFP

Filtering Face Piece

\section{Declarations}

\section{Ethics approval and consent to participate}

The data of patients used in this study were part of routine clinical practices in the Amphia hospital and their anonymousous use is beyond the scope of the Medical Research Involving Human Subjects Act (MRIHS). The use of the data of Health care workers were reviewed by the Ethics Committee Brabant, the 
Netherlands (METC Brabant/20.134/NW2020-26). This was also judged to be beyond the scope of the MRIHS and a waiver of written informed consent was granted.

Consent for publication

Not applicable

Availability of data and materials

The datasets used and/or analysed during the current study are available from the corresponding author on reasonable request.

Competing interests

The authors declare that they have no competing interests

Funding

Not applicable

Authors' contributions

$\mathrm{JK}, \mathrm{WvdB}$ and MvR designed the study. VW wrote the manuscript with input from all authors. MT performed the calculations for nosocomial transmission in patients. JM performed the calculations of sick reports among employees. All authors provided critical feedback and helped shape the research, analysis and manuscript.

\section{References}

1. Corman Victor M, Landt Olfert K, Marco MR, Adam M, Daniel C. KW, et al. Detection of 2019 novel coronavirus (2019-nCoV) by real-time RT-PCR. Euro Surveill. 2020;25(3):pii = 2000045. https://doi.org/10.2807/1560-7917.ES.2020.25.3.2000045.

2. $10.1101 / 2020.03 .23 .20041913$

Marjolein Kluytmans A, Buiting S, Pas. Robbert Bentvelsen, Wouter van den Bijllaardt, Anne van Oudheusden, et al. medRxiv 2020.03.23.20041913; doi: https://doi.org/10.1101/2020.03.23.20041913.

3. Lauer SA, Grantz KH, Bi Q, et al. The Incubation Period of Coronavirus Disease 2019 (COVID-19) From Publicly Reported Confirmed Cases: Estimation and Application. Ann Intern Med. 2020 May;5(9):577-82. doi:10.7326/M20-0504. Epub 2020 Mar 10. 172 ) .

\section{Figures}




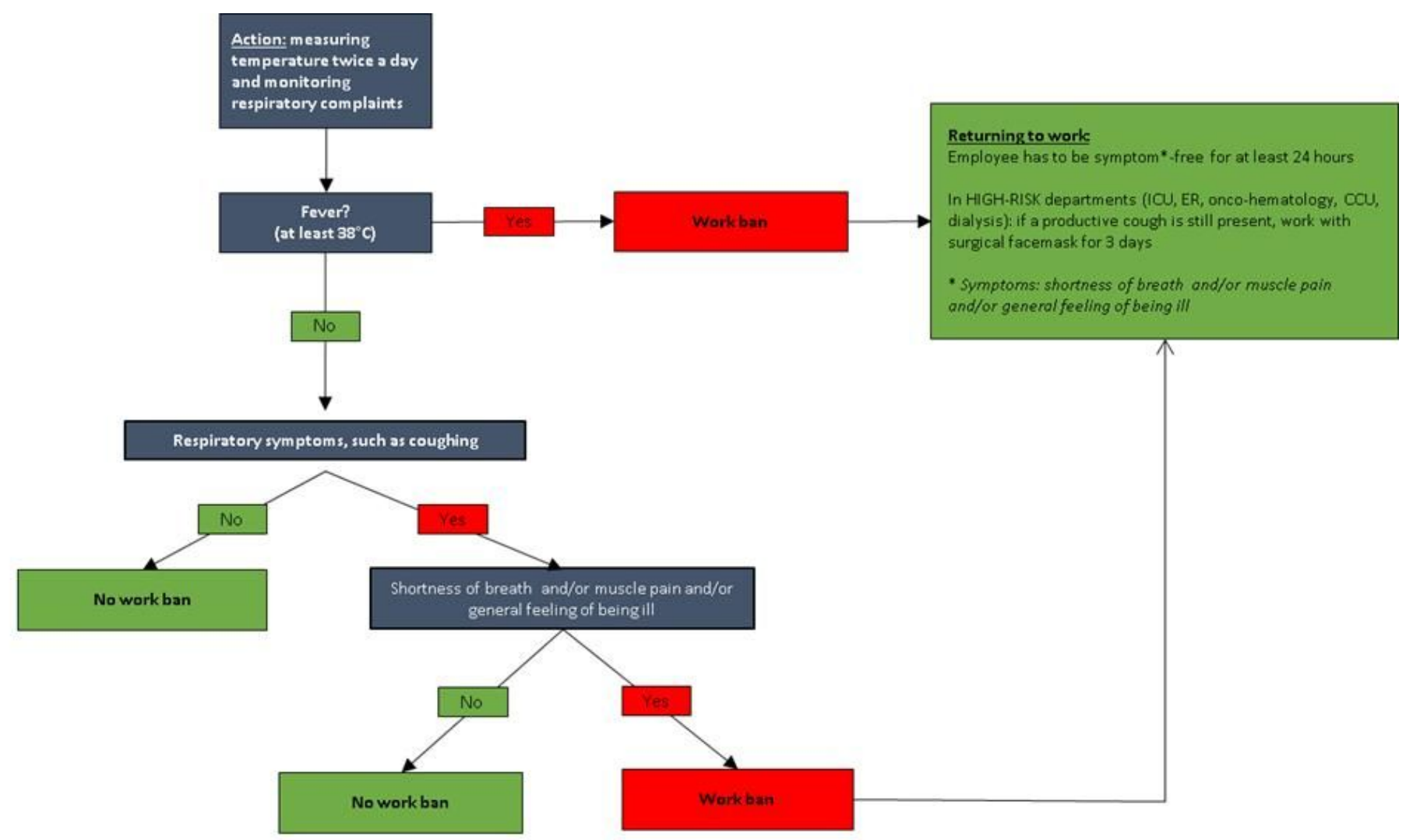

Note: If it concerns an employee where the absence leads to disruption of continuity of critical processes, a test can be considered. This test is only used after approval by the business manager / medical manager of the department concerned.

\section{Figure 1}

flowchart to determine if an employee is permitted to continue work based on symptoms only

(a) Total number of sick reports, per week (2020)

(b) Total number of sick reports in the first 20 weeks

Period of active testing employees

(2017-2020)

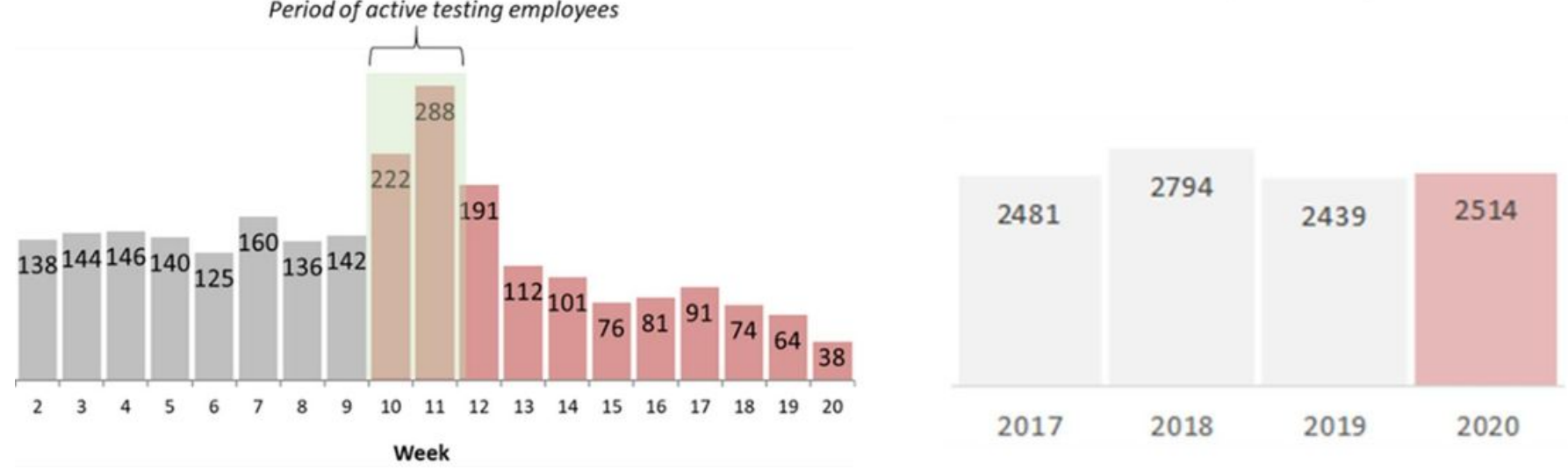

Figure 2

shows the development of the number of sick reports per week in 2020. In week 10 (march 3), active testing of employees for COVID-19 was started (green frame). From the end of week 11 (March 13), the 
decision if an employee is permitted to continue work was fully based on symptoms without a test being performed. Figure $2 \mathrm{~b}$ shows the total number of sick reports in the period from week 1 to week 20 , from 2017 through 2020.

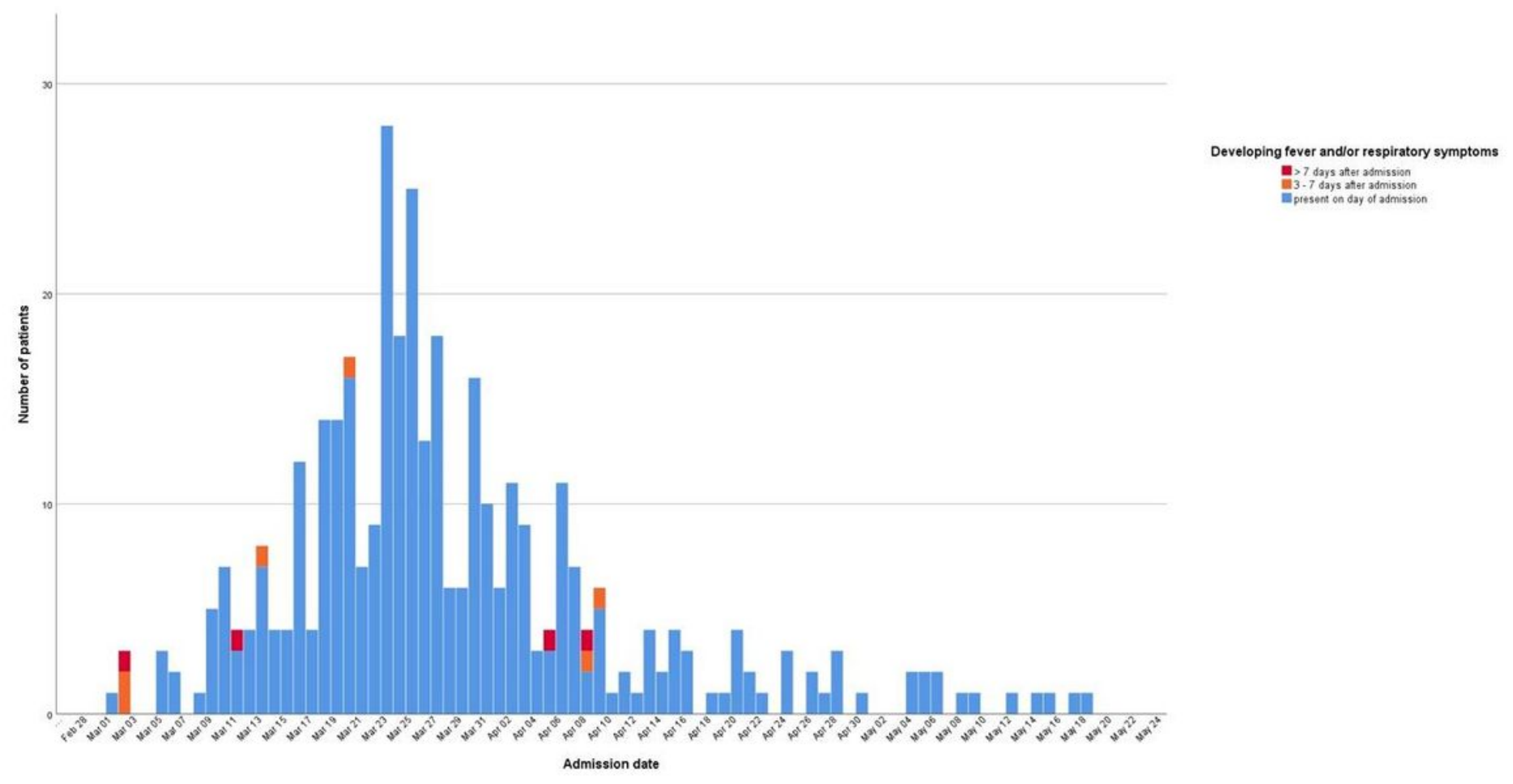

Figure 3

shows the number of COVID-19 positive patients admitted to the hospital in relation to the day of developing fever and/or respiratory symptoms after the admission date. The green box represents the period when employees were actively tested for SARS-CoV-2 according to the flowchart. 